\title{
Computational representation and hemodynamic characterization of in vivo acquired severe stenotic renal artery geometries using turbulence modeling
}

\section{George C Kagadis ${ }^{1}$, Eugene D Skouras ${ }^{2,3}$, George C Bourantas ${ }^{1}$, Christakis A Paraskeva $^{2,3}$, Konstantinos Katsanos ${ }^{4}$, Dimitris Karnabatidis ${ }^{4}$ and George C Nikiforidis $^{1}$}

${ }^{1}$ Department of Medical Physics, School of Medicine, University of Patras, GR 26500, Rion, Greece

${ }^{2}$ Department of Chemical Engineering, University of Patras, GR 26500, Rion, Greece

${ }^{3}$ Institute of Chemical Engineering and High Temperature Chemical Processes - Foundation for Research and Technology, P.O. Box 1414, GR-26504, Patras, Greece

${ }^{4}$ Department of Radiology, School of Medicine, University of Patras, GR 26500, Rion, Greece

E-mails: george.kagadis@med.upatras.gr,gnikif@med.upatras.gr

\begin{abstract}
The present study reports on computational fluid dynamics in the case of severe renal artery stenosis (RAS). An anatomically realistic model of a renal artery was reconstructed from CT scans, and used to conduct CFD simulations of blood flow across RAS. The recently developed Shear Stress Transport turbulence model was pivotally applied in the simulation of blood flow in the region of interest. Blood flow was studied in vivo under the presence of RAS and subsequently in simulated cases before the development of RAS, and after endovascular stent implantation. The pressure gradients in the RAS case were many orders of magnitude larger than in the healthy case. The presence of RAS increased flow resistance, which led to considerably lower blood flow rates. A simulated stent in place of the RAS decreased the flow resistance at levels proportional to, and even lower than, the simulated healthy case without the RAS. The wall shear stresses, differential pressure profiles, and net forces exerted on the surface of the atherosclerotic plaque at peak pulse were shown to be of relevant high distinctiveness, so as to be considered potential indicators of hemodynamically significant RAS.
\end{abstract}


Keywords: Computational fluid dynamics, hemodynamics, renal artery stenosis, ComputerAided Therapeutic Planning, Computed Tomography, segmentation, simulated reconstruction, turbulent flow.

\section{Introduction}

Renal artery stenosis (RAS) afflicts patients with atherosclerotic disease and leads to renal malperfusion, systemic hypertension, and ischemic nephropathy [1]. It is ranked as mild, moderate, and severe RAS according to the degree of stricture of the vessel diameter. The gold standard method for the assessment of RAS is intra-arterial Digital Subtraction Angiography (DSA), although modern multidetector CT systems (MDCT) are gradually being used instead, due to their non-invasive nature. The only available functional indicator for hemodynamically significant RAS (severe stricture or a moderate one with a pressure gradient of at least $5-10 \%$ ) is the renal artery differential pressure (RADP), which is measured with the use of a catheter [2-4]. However, it is not widely used because it involves catheterization of the target vessel and its correlation with the outcome of the elected therapy is disputed $[5,6]$.

Turbulence consists of fluctuations in the flow field in time and space. It is a complex process, mainly because it is three dimensional, unsteady and consists of many scales. It can have a significant effect on the characteristics of the flow. Turbulence occurs when the inertia forces in the fluid become significant compared to viscous forces, and is characterised by a high Reynolds number. Turbulence may be observed along vessel stenoses, depending on the local geometry and physical conditions, and influences blood flow. It affects the flow resistance, shear stress, and mass transport on the vessel wall, tensile stress on the endothelial cell membrane, hemostasis, and cell proliferation and migration $[7,8]$. In experimental studies of pulsatile flow through constricted tubes [9], vortical and helical structures were observed within mild stenoses. Transition to turbulence was associated with the breakdown of waves, and streamwise vortices occurred at these stenoses. Hemodynamic parameters such as pressure, velocity, and shear stress are spatially and temporally dependent. Scientific evidence underlines the significant role of wall shear stress (WSS) in the localization and progression of atherosclerotic plaque, and in the development of neointimal hyperplasia after endovascular stenting [10-13]. Plaque encroachment into the lumen induces shear stress variations along the vessel wall, affecting both disease progression and renal perfusion [14, $15]$. 
Currently, there is a clinical demand for non-invasive screening tests of RAS that could simultaneously perform morphological and functional evaluation of RAS, and focus on proper patient selection for either conservative or interventional treatment. Computational fluid dynamics (CFD) modeling can be used to non-invasively study blood velocity and pressure within the vessel lumen, test the hypotheses of disease formation, and evaluate novel intravascular devices prior to in vivo implementation [13]. Recent studies investigating blood flow in phantom models and real stenotic arteries underscore the significant impact of local mass transfer and shear stress forces in the pathogenesis and natural course of atherosclerosis [16-19]. It also stresses the necessity of focal, patient-specific hemodynamics to be elaborately characterized, especially prior to interventional therapy. Realistic estimation of RADP with acceptable accuracy by CFD has already been reported [4].

The present study focuses on the computational simulation and calculation of hemodynamic properties, aiming to expand CFD into functional and prognostic assessment of RAS before and after endovascular therapy. The blood flow in a human renal artery is examined under the presence of an atherosclerotic plaque at the ostium of the vessel. The simulated cases of a healthy vessel and an atherosclerotic vessel after stent implantation are analyzed as well. The flow field, local and overall pressure gradients, shear stresses, and resultant forces exerted on the atherosclerotic plaque are meticulously investigated. In this concept, the $k-\omega$ based Shear Stress Transport model [20-22], is applied on raw CT imaging data in order to simulate the flow and critical flow properties as well as the hemodynamic parameters of RAS under in vivo conditions.

\section{Materials and Methods}

\subsection{Data acquisition and segmentation}

A patient with severe RAS underwent contrast-enhanced spiral CT (Siemens, Somatom Plus 4A). The examination protocol was set to $120 \mathrm{kVp}, 180 \mathrm{mAs}$, with concurrent administration of $140 \mathrm{ml}$ non-ionic iodinated contrast agent at $3.5 \mathrm{ml} / \mathrm{s}$ (time delay was set to $16 \mathrm{~s}$ ). Scanning coverage was $6 \mathrm{~cm}$ with $2 \mathrm{~mm} / \mathrm{s}$ table feed (total examination time was $30 \mathrm{~s}$ ) and reconstruction was performed with a soft-reconstruction kernel at $1 \mathrm{~mm}$ intervals. The acquisition protocol generated a stack of axial images. Data were first archived to a DICOM server and transferred to a PC where image processing was performed, in order to generate the three-dimensional objects of interest. 
Initially, all DICOM files were used to form a three-dimensional volume. The raw DICOM files were loaded into the 3D image processing software Analyze 6.0 (www.analyzedirect.com), and a 3D reconstruction of the vessel lumen surface was produced. Thresholding and seeded region growing techniques were applied subsequently in order to segment the renal arteries and the aorta. The triangulated surface was then exported in a format that was 'understandable' by the CFD software (STL format) (Fig. 1). A generalpurpose algorithm based on an adaptive mesh model was used. This algorithm is based on previous work [23], and is an embedded function to Analyze 6.0 software [24].

\subsection{Surface editing and treatment of segmented data}

The STL segmented data obtained from the imaging software were fully imported into the advanced mesher, where all the segments of irrelevant surface portions were cut-off -- leaving only the ones representing the renal arterial segment. In order to maximize the discretization capability at the place of the RAS, boundary conditions (BC) were applied exclusively to the renal artery without extension of the calculation domain into the aorta, in order to avoid increase in the computational load (Fig. 2 a,b). However, realization of the aorta and simulation of the blood flow within was performed on model geometries for validation purposes.

Additional inlet and outlet surfaces were properly mounted at each opening of the arterial segment, with normal surface along the main flow direction. These inlet and outlet mouths were carefully attached to the respective wall segments and tightly tailored to the adjacent wall STL surfaces, in order to have consistent representation of the blood flow. The complexity of this structure was high at the inlet mouth, because plaque development twisted the wall geometry from the cylindrical form (Fig. 2 a,b). Fine surface mesh resolution near the inlet is shown in Fig. 3 [25]. Simulation results using these reconstructed data are reported as Case 1. The areas of all distinct surfaces of Case 1 are given in Table 1.

The arterial wall regions at the atheromatic plaque are named "Plaque" in all Tables and Figures, and can be seen as blue regions on Fig. 4 and 5. Resistance to flow through the "Plaque" area was largely increased due to the three constrictions that were left open to flow by the formation of the atheromatic plaque. One of the constrictions had minimum size calculated average ( $\mathrm{ca}$ ) $0.46 \mathrm{~mm}$, while the sizes of the other two were $0.6 \mathrm{~mm}$. In comparison, the mean distance between inlet and outlet fronts is approximately $35 \mathrm{~mm}$. The 'Plaque' area is defined as the minimum number of STL segmented wall surfaces that (i) are 
fully affined to the inner surface of the plaque; (ii) fully include the adjacent constricted lumen; (iii) form a convex shape; and (iv) the boundary intersection between this area and the adjacent arterial wall forms a closed curve, with the tangent along it maintained perpendicular to the flow direction. Small alterations on the final "Plaque" shape lead to negligible deviations on the results of average and maximum WSS, as long as the stenosed areas are included. Calculations on a plaque area $10 \%$ extended in both directions along the main flow resulted in less than $1 \%$ modification of average shear stress and no alteration in the maximum value.

\subsection{Simulation of unobstructed cases}

In the absence of alternative segmented data of the same patient, and in order to simulate a stent placement at the stenosis, the arterial walls of Case 1 were further manipulated. The STL wall surfaces were sharply cut by a plane at a suitable point, and were replaced by a model cylindrical segment. The purpose of this cylindrical segment that extended from the cutting plane to the arterial inlet mouth, was used as an idealized arterial segment achieved after stent implantation (Fig. 4 b,d). The cylindrical substitute that simulated the stent was of equal axial length as the cut-off plaque segment and of equivalent radius as the rest of the artery. The same boundary and physical conditions of Case 1 were applied to the 'post-treatment' case, defined as Case 2.

In order to completely describe the clinical problem, the healthy renal artery of the same patient was simulated. Therefore, the RAS segment of Case 1 was replaced by a cylindrical model segment. Contrary to Case 2, the circular base drawing of the cylindrical section initiated from the arterial inlet (Fig. 4 b,f). The continuity and the smoothness of the arterial surface were significantly improved, compared to the sharp intersection of the respective parts in Case 2. Results were collected using the same CFD conditions that were applied to Cases 1 and 2, and data relative to this 'healthy' formation are listed as Case 3. For simplicity, the replaced arterial portion of each unobstructed case was also labeled "Plaque". The areas of all individual surfaces of Cases 2 and 3 are reported in Table 1.

\section{$2.43 D$ meshing method}

The ANSYS CFX (v5.7) commercial package was used for mesh generation. The model simulations applied an Octree-based meshing algorithm in order to fill the interstitial 
segmented arterial surfaces with tetrahedral or prismatic cells. Prescribed curves and points on the surface mesh were used to adjust the positions of edges and vertices in the volumetric mesh. Initial discretizations include a minimum of 10 elements across an individual artery cross-section [23, 25-28]. Final discretization includes multiple values of the initial resolution, typically on the order of 15 elements across the cross-section of the minimum arterial constriction (Fig. 3). This is crucial to the procedure, as fine discretizations near the atheromatic plaque regime are necessary for the accuracy of local differential pressures and WSS measured thereat. An analysis of the mesh dependence and of the effect of the discretization level on the process is given in the "Cases Studied" section.

\subsection{Computational Fluid Dynamics}

Under normal rest conditions, the change of vessel diameter during the cardiac cycle is observed to be less than $10 \%$ in most of the major arteries [29-31]. In atherosclerosis, the arteries are less compliant and the wall motion is reduced. Thus, the vessel walls were considered to be rigid and the assumption of zero wall motion was used for the calculations. The size of the suspended red blood cells does not affect blood flow noticeably in typical flow conditions. If blood density is not altered considerably due to high glucose levels or blood irregularities, blood viscosity is expected to cause negligible variations on the flow configuration. It is still an open subject whether blood can be simulated successfully by purely a Newtonian fluid. Recent two phase models (where hematocrit can build up) showed the contrary. However, in the present study, blood was treated as an incompressible, single phase, Newtonian, and isothermic fluid in a fixed domain with non-slipping wall boundaries with reasonable accuracy $[17,18]$. The formulation of the problem consists of the continuity (mass) and Navier-Stokes (momentum) equations, and suitable initial and boundary conditions. The finite element method (ANSYS CFX) was employed for solving the transient equations in the complex domains of this type.

$\underline{\nabla}(\rho \underline{\mathbf{v}})=\underline{\mathbf{0}}$

$$
\frac{\partial(\rho \underline{\mathbf{v}})}{\partial t}+\underline{\nabla} \square(\rho \underline{\mathbf{v}} \underline{\mathbf{v}})=\underline{\nabla}\left(p+\frac{2}{3} \rho k\right)+\underline{\nabla}\left(\mu_{e f f}\left(\underline{\nabla} \underline{\mathbf{v}}+(\underline{\nabla} \underline{\mathbf{v}})^{\mathrm{T}}\right)\right)
$$


Turbulence models seek to solve a modified set of transport equations by introducing averaged and fluctuating components. For example, a velocity component may be divided into an average component, and a time varying component. In general, turbulence models seek to modify the original unsteady Navier-Stokes equations by the introduction of averaged and fluctuating quantities to produce the Reynolds Averaged Navier-Stokes (RANS) equations []. These equations represent the mean flow quantities only, while modelling turbulence effects without a need for the resolution of the turbulent fluctuations. In an 'eddy viscosity' description, turbulence consists of small eddies which are continuously forming and dissipating, and in which the Reynolds stresses are assumed to be proportional to mean velocity gradients. Twoequation eddy viscosity models, such as the $k-\varepsilon$ and the $k-\omega$ models, are very widely used for the description of turbulence, as they offer a good compromise between numerical effort and computational accuracy. Both the velocity and length scale are solved using separate transport equations. The $k-\varepsilon$ and $k-\omega$ two-equation models use the gradient diffusion hypothesis to relate the Reynolds stresses to the mean velocity gradients and the turbulent viscosity. The turbulent viscosity is modelled as the product of a turbulent velocity and turbulent length scale. The turbulence velocity scale is computed from the turbulent kinetic energy, which is provided from the solution of its transport equation. The turbulent length scale is estimated from two properties of the turbulence field, usually the turbulent kinetic energy and its dissipation rate. The dissipation rate of the turbulent kinetic energy is provided from the solution of its transport equation.

$$
\begin{aligned}
& \mu_{e f f}=\mu+\mu_{T U R B}, \mu_{T U R B}=0.09 \frac{\rho k^{2}}{\varepsilon} \\
& \frac{\partial(\rho k)}{\partial t}+\underline{\nabla} \varphi(\rho \underline{\mathbf{v}} k)=\underline{\nabla}\left[\left(\mu+0.09 \frac{\rho k^{2}}{\varepsilon}\right) \underline{\nabla} k\right]+P_{k}-\rho \varepsilon \\
& \frac{\partial(\rho \varepsilon)}{\partial t}+\underline{\nabla}\left[(\rho \underline{\mathbf{v}} \varepsilon)=\underline{\nabla}\left[\left(\mu+\frac{0.09}{1.3} \frac{\rho k^{2}}{\varepsilon}\right) \underline{\nabla} \varepsilon\right]+\frac{\varepsilon}{k}\left((1.44) P_{k}-(1.92) \rho \varepsilon\right)\right.
\end{aligned}
$$


$\mu_{\text {eff }}=\mu+\mu_{T U R B}, \mu_{T U R B}=\frac{\rho k}{\omega}$

$\frac{\partial(\rho k)}{\partial t}+\underline{\nabla}\left[(\rho \underline{\mathbf{v}} k)=\underline{\nabla}\left[\left(\mu+\frac{1}{2} \frac{\rho k}{\omega}\right) \underline{\nabla} k\right]+P_{k}-(0.09) \rho k \omega\right.$

$\frac{\partial(\rho \omega)}{\partial t}+\underline{\nabla}(\rho \underline{\mathbf{v}} \omega)=\underline{\nabla}\left[\left(\mu+\frac{1}{2} \frac{\rho k}{\omega}\right) \underline{\nabla} \omega\right]+\frac{5}{9} \frac{\omega}{k} P_{k}-(0.075) \rho \omega^{2}$

The Shear Stress Transport (SST) model was adopted here, which is a superset of the standard $k$ - $\omega$ model, and is more accurate and robust in overcoming near-wall treatment errors for lowReynolds number computations. This model accounts for the transport of the turbulent shear stress by the inclusion of transport effects into the formulation of the eddy viscosity. The main problem with the standard $k-\omega$ model is its strong sensitivity to freestream conditions. To this end, a transformation (blending) between the $k$ - $\omega$ model near the surface and the $k$ - $\varepsilon$ model in the outer region was developed for the description of the wall functions [20-22]. It consists of a transformation of the $k-\varepsilon$ model to a $k-\omega$ formulation and a subsequent addition of the corresponding equations. The k- $\omega$ model is thereby multiplied by a blending function $F_{1}$ and the transformed $k-\varepsilon$ model by the function $\left(1-F_{1}\right) . F_{1}$ is equal to one near the surface and switches over to zero inside the boundary layer (i.e. a function of the wall distance). The corresponding $k$ - and $\omega$ - equations are added to give the SST model at the boundary layer edge and outside the boundary layer, the standard $k-\varepsilon$ model is therefore recovered. The blending functions are critical to the success of the method. Their formulation is based on the distance to the nearest surface and on the flow variables.

$F_{1}=\tanh \left(\max \left(\frac{2 \sqrt{k}}{0.09 \omega y}, \frac{500 v}{y^{2} \omega}\right)^{2}\right)$,

where $y$ is the distance to the nearest wall and $v$ is the kinematic viscosity. These additions provide highly accurate predictions of the onset and amount of flow separation and recirculation under adverse pressure gradients at arterial stenoses. [20-22] A comparison of the SST performance in hemodynamics to the performance of older two-equation turbulence models, such as the $k-\varepsilon$ and the $k$ - $\omega$ families of models, may be relevant to further validation of the reported results $[32,33]$.

\subsection{Cases Studied}

Case 1: Renal Artery segment with atheromatic plaque at flow entrance (reconstructed).

Case 2: Renal Artery segment with stent implantation and no atheromatic plaque (simulated).

Case 3: Renal Artery segment with no atheromatic plaque - healthy individual (simulated). 
Transient mass balance equations were solved with 1/100th of a second temporal resolution and a spatial resolution starting from $12 \mu \mathrm{m}$ [25]. Computations of the stenosed renal artery are reported using a fine mesh of 632250 nodes between 3434237 finite elements. Reproducibility and convergence were checked by performing the same simulations on a less fine mesh of 455366 nodes and 2593145 elements with the same time step (error in average WSS $\sim 4 \%$ ), in addition to calculations using the finest step and a temporal resolution of $0.025 \mathrm{~s}$ (error in WSS $\sim 7 \%$ ). The fine computation domain was partitioned in half using a MeTiS multilevel weighted k-way algorithm (CFX manual[34]). Simulations were performed parallelized (message passing techniques, 4 nodes) on modern high-end hardware (Dual Xeon), affordable by typical clinical laboratories, where the calculations of each simulation purposively last no more than $1 \frac{1}{2}$ day. The order of magnitude of the less fine spatial mesh was found to be adequate for the other two cases; thus, 2163189 elements were used for Case 2, and 2012162 elements for Case 3; both latter Cases use a $0.025 \mathrm{~s}$ time step.

Flow entry and exit from the calculation domain was assumed to be normal to the inlet and outlet surface regions of the arterial geometries (Table 1, Fig. 4 and 5). Moreover, flow was allowed to reenter through any part of the inlet and outlet region during a pulse, i.e., opening conditions instead of flow entry only or flow exit only BCs. The total pressure values of the inlet and the outlet faces, averaged over each face, were fixed at each time step. Data for the overall average pressure drop and corresponding volumetric flow through the boundaries of idealized renal arteries have been reported and are reasonably sufficient as input to the present calculations $[4,17,35]$. The sinusoidal approximation, described by Yim et al $[4,36]$, was applied for the boundary pressure pattern description in all cases. In the sinusoidal approximation, description of the mean pressure drop evolution between the inlet and outlet of the arterial segment is in the form:

$$
P(t)=P_{0}+P_{\max } \sin (\varpi t)
$$

with $P_{0}, P_{\max }$, and $\varpi$ taken from experimental renal data from Yim et al [4]. The temporal evolution of the pressure drop of Case 1 was directly extracted from this work -- in the absence of in-house or other published validated data for this case. The value of the pressure drop represents the pressure head necessary to drive blood flow to the distal arterial branches [25]. The pressure drop evolution of Case 2 refers to a stent-implanted situation of the previous case (by Percutaneous Transluminal Angioplasty), where no similar data could be extracted from the literature.

It was assumed that the kidney would maintain constant blood flow (i.e. equal mean pressure drop per pulse between the arterial inlet and outlet) in all cases as it works under constant 
mean pressure drop conditions. Under this assumption, perfusion equilibrium between the aorta and the distal renal parenchyma was either compensated by systemic hypertension prior to intervention, or completely restored after virtual implantation of a stent, as the one simulated in Case 2. For the implementation of comparison, Case 3 (healthy state) was considered with the same BC. Thus, the same boundary mean pressure drop pattern description of Eq. 1 with identical values for $P_{0}, P_{\max }$, and $\varpi$, was applied in the three reported cases. For validation purposes, realizations and simulations of the aorta were performed. Comparative results using this model against experimental literature data [4] are given in the Results section.

\section{Results}

\subsection{Hemodynamic parameters}

Data presented in Fig. 6, 7, and 8 were obtained from post-processing of the transient simulation runs in each case. Due to the large amount of data obtained during each transient run, a complete set of hemodynamic data and flow properties were retrieved and postanalyzed every $0.1 \mathrm{~s}$ (10 time steps) in a complete pulse cycle, while splines were drawn over the points in the graphs shown. Blood flow rates in the model renal artery are shown in Fig. 6. The changes in local wall curvature of the artery in all cases cause a significant deviation from the results of a model cylindrical tube of equal radius. The presence of the atheromatic plaque in Case 1 caused a considerable additional resistance to flow compared to the open channel geometry of Cases 2 and 3, where no plaque is present. This led to considerably lower renal artery flow rates in Case 1 compared to the other two Cases. Local Reynolds number, however, based on the constriction size, was found to reach values greater than 800 in the stenosis of Case 1.

The local values at each point of the inlet and outlet fronts differ as the reference pressure values imposed at each time step of the pulse (Eq.1). The pressure is calculated at each point and the average value over the area of each front is obtained. The difference of the average pressures between the two fronts converges to the reference value of the particular time step by repeated iterations. No significant deviations in average pressure drop profiles during a pulse were calculated between Cases 2 and 3 (Fig. 7 b,c). However, mean and maximum pressure profiles were higher in Case 1 (Fig. 7a), where plaque presence caused increased flow resistance. The arterial stenosis caused a diffusion of the local pressure gradient on the surface of the inlet front, showing same values of differential pressure in both maximum and average ranges at all time steps within a pulse. The presence of a stent in the artery, Case 2, 
evoked a maximum pressure drop almost equal to the average one. The flow within a cylindrical stent of this kind is generally more homogeneous, compared to the flow in a curved vessel of a healthy patient, Case 3 . Thus, variation of the local pressure gradient on the inlet front in presence of the stent is limited. Comparison of average differential pressure profiles among the three Cases document the additional resistance to blood flow due to the plaque (Fig. 7d).

Peak values of maximum WSS on the surface of the plaque in Case 1 were almost an order of magnitude higher than corresponding average ones, located at the narrowest part of the arterial stenosis (Fig. 8a). A healthy artery also exhibits a high maximum-to-average ratio of WSS values at certain points in the plaque area, however, of much lower maximum WSS magnitude compared to Case 1 (Fig. 8c). On the contrary, the stent apparently acts as a diffuser on the distribution of WSS along the arterial wall (Fig. 8b). Maximum WSS values and maximum-to-average WSS ratio are reduced. A comparison of average WSS values of the cases studied is shown in Fig. 8d. Average WSS at peak flow along with the resulting forces acting on the plaque area are given in Table 2.

Points along selected streamlines at peak flow, as shown in Fig. 5, were chosen randomly for each case. The local relative pressure and pressure gradient along the spatial coordinate (X) were post-calculated and reported in Fig. 9. X was the local axis that lies along the axial coordinate of the main part of the artery (cf. Fig. 4); this direction was practically normal to the inlet flow surfaces of the non-stenosed cases (Case 2 and 3) and it would be the local axis of rotation if the arterial geometry was assumed ideally tubular. Differences between the stenosed artery case (Case 1) and each of the unobstructed cases (Cases 2, 3) are of many orders of magnitude and are clearly distinct. This information also exhibits the potential of the applied CFD methodology to non-invasively predict the effect of RAS development on the spatiotemporal flow and pressure distribution throughout an arterial segment.

\subsection{Validation}

In order to evaluate the model's accuracy, a comparison with an identical model geometry described by Yim et al [4] was conducted. Model geometry of the RAS and the adjacent aorta were reconstructed from scratch (Fig. 10). The BC used in Cases 1-3 were applied to this geometry, using the present CFD methodology. Transient simulation results of a complete pulse cycle with a $0.0125 \mathrm{~s}$ temporal resolution were collected and compared to the literature data. In order to study the effect of different discretization on the accuracy and convergence of the results, two individual mesh discretizations were used. The finest mesh discretization 
applied was 173870 nodes and 925630 elements, with the height of the smallest element at approximately $13 \mu \mathrm{m}$. This element was located in the narrowest constriction, next to the plaque wall. The element height at the constricted regions was exponentially increased in the direction normal to the walls, forming up to five (prismatic) layers, in order to handle the turbulence boundary layer efficiently. The total volume of the configuration was calculated at $255.616 \mathrm{~mm}^{3}$. The second discretization had a coarser resolution; 49408 nodes, 242071 elements, approximately $20 \mu \mathrm{m}$ the smallest element size, and twice the temporal resolution $(0.0250 \mathrm{~s})$. Accuracy between post-processed results of the two configurations at peak flow was measured as the difference (in percentage) of the two resolutions (Table 3).

Differential pressure values of a complete pulse cycle using the finest discretization model, as well as the respective literature values, as functions of the flux inside the stenosed arterial segment, are shown in Fig. 11. Compliancy with the reference data is apparently adequate in the lower flow curve (lower region of the loop). The error for low flow regimes (flux $<25 \mathrm{cc} / \mathrm{s}$ ) is minimal between the present calculations and Yim et al[4]; the percent error between the two simulation results is approximately $6.7 \%$ at $21.8 \mathrm{cc} / \mathrm{s}$ (curves 1 and 3 ). Moreover, the experimental results are represented more efficiently by the present computations at near-peak flow (flux $>25 \mathrm{cc} / \mathrm{s}$ ); error approximately $6.2 \%$ at $26.4 \mathrm{cc} / \mathrm{s}$ between these simulations and Yim et al (curves 2 and 3). The hysteresis (upper region of the loop) of the present simulations is attributed to the turbulence effects of the pulsatile flow and the relative flow reversal phenomena that were observed within stenosed arteries. This effect was not explicitly addressed by Yim et al [4].

Additional studies using (non-stenosed) segmented data in the renal region of a patient uncorrelated to the Cases 1-3, which had relevant hemodynamic properties measured by external ultrasound (US), was performed for validation purposes. The whole aorta-arterial segment was also acquired by CTA and was consequently segmented and meshed in this case, as there were no constricted regions present to increase the local discretization level significantly. The same degree of accuracy and convergence was kept as in Cases 1-3. After cutting off irrelevant segmented elements and tailored drawing of inlet and outlet boundaries at the open faces (Fig. 12a), simulations of blood flow were performed in a meshed spatial domain of 245324 elements. The measured data refer to mean volumetric rates and maximum (systolic) and minimum (diastolic) velocity profiles in certain points in the arteries, and are given in Table 4. In the absence of pressure profile data measured in this case, steady state calculations were applied in order to reproduce the measured volumetric rate and velocity data in an averaged fashion. To this end, the volumetric rates measured on the orifices of the 
two renal arteries were applied as $\mathrm{BC}$ in the respective regions (distal to aorta). The aim was to reproduce and compare the mean velocity components proximal to aorta between pulses and, thus, compare the accuracy of the modeling. The BCs in the inlet and outlet part of the renal aorta were not known, and were estimated as follows. The volumetric rate at the inlet part of the aorta was set either to an order of magnitude, i.e. ten times larger than the sum of the volumetric rates of the two renal arteries (Case A), or to half the former value, i.e. five times larger (Case B). The boundary at the outlet of the aorta was set to zero relative pressure at all times. Simulation of the blood flow was performed using SST turbulence modeling. Streamlines taken after post-processing of Case B are depicted in Fig. 12b. Simulation results of both cases are given in Table 4, and render the behavior of the velocity profiles that fall well within the measured range of both renal arteries, regardless of the $\mathrm{BC}$ used for the aorta inlet. Table 4 also displays the ratio of the volumetric rate calculated after convergence to the volumetric rate originally imposed (which is equal to the measured value) to the distal outlet parts of each artery for each case.

\section{Discussion}

The present study focused on imaging and evaluation of RAS, which is the main cause of renovascular hypertension (RVH) [2, 3]. Atherosclerotic RAS is typically caused by ostial plaque formation with an eventual occlusion rate up to $18 \%$ over 5 years [37]. Apart from causing renal hypoperfusion and loss of kidney filtration capacity, RAS is currently considered to be an independent predictor of death regardless of the extent and severity of systemic atherosclerosis [38]. Given the clinical significance of RAS, the frequent inconclusive nature of translesional pressure gradients for functional assessment of RAS, and the consensus that RAS revascularization should be ideally performed even before clinical evidence of renal malfunction, it is obvious that a reliable, accurate and reproducible noninvasive screening test for RAS is mandatory $[38,39]$. To this aim, the authors pursued a realistically elaborate blood flow distribution and regions of recirculation, pressure gradients, and areas of high and low wall shear stress within the stenotic vessel segment by modern turbulence-based CFD modeling.

The majority of published CFD reports mainly deal with MR, which is radiation free. However, in daily clinical practice, many studies are performed under CT imaging. Modern multi-detector CTs offer images of high spatial and temporal resolution, and may suppress the motion artifacts due to breathing and artery pulsation. The process of generating CFD models from CT images is still quite complex, but more user-friendly image processing software has 
emerged. In the present study, anatomically realistic models of renal arteries were constructed from CT scans and used to conduct CFD simulations of blood flow throughout the renal artery.

Recent literature reports have provided measures of WSS and differential pressures across stenoses [4]. Other groups have investigated the relationship between shear stress magnitude and development of atherosclerotic plaque by applying laminar flow CFD models [40, 41]. Numerical studies on steady and pulsatile flow through stenotic vessels raise several turbulence issues, including fluid-structure interaction and rheology effects. [42] [43-45] [46] [47]. To date, no known studies incorporating turbulence have focused on the forces and the WSSs that are exerted on the atherosclerotic plaque, along with flow properties of segmented geometries. The current study is one of the first to simulate blood flow across a stenosed segment due to plaque encroachment, which did not use any invasive means. The mainstream therapy of stent placement was also simulated so as to virtually portray the restoration of vessel patency and normalization of the flow. Enhanced wall functions were employed along with the turbulence model in order to better resolve the near wall region using the Shear Stress Transport formulation [20-22]. These functions are better suited for lower Reynolds number flows with complex near-wall phenomena. However, this process is computationally more intensive as it requires a very fine near wall mesh. This is the major motivation for not including the aorta region in the present calculations.

The major goal of applying CFDs in Simulation-Based Medical Planning is to spot potential indicators for therapeutic intervention and prognostic criteria for its outcome. It is noteworthy that published data regarding RAS management show a significant discordance between successful revascularization procedures $(>95 \%)$ and an improvement in hypertension and renal perfusion (up to $70 \%$ ) $[38,39,48]$. These data reasonably reflect the absence of a proper and efficient test for combined anatomic visualization and functional evaluation of RAS. To this end, CFD models may represent an attractive alternative. The present analysis included calculation of the flow rate, overall pressure drop, wall shear stress and wall shear stress rate, along with the internal pressure profile across the artery at peak pulse. These variables are considered the most prominent diagnostic and prognostic indicators of the patient condition. Among these indicators, the high ratio of maximum-to-average WSS (i.e., contrast-to-noise), in conjunction with the high peak values of the maximum WSS and the differential pressure at the point of stenosis, may be presumed to be highly distinctive quantitative parameters of the clinical condition of the patient. 
Theoretically, the above parameters may be calculated first upon diagnosis of RAS in order to determine severity, and secondly after virtual stent placement in order to assess the outcome prior to real endovascular therapy. Since progression of atherosclerosis and neointimal hyperplasia after stent placement are affected by the local WSS fluctuations, future exciting goals would be to predict the risk and rate of these phenomena by CFD-based characterization and simulation.

An identified limitation of the present work is the inclusion of a single patient and the absence of corresponding actual validation data. However, the high-grade of the lesion under study prohibited selective catheterization and translesional pressure drop measurements. The primary focus of this study was the methodological approach of turbulence-based computerized modeling of RAS hemodynamics. The assumption of rigid vessel walls is expected to influence the estimation of the proposed parameters. Nevertheless, this limitation has been reported to have minimum effect in rest conditions in atherosclerotic vessels [29-31].

Based on clinical data from large-scale trials, quantitative prognostic markers and evidencebased indicators could be standardized and proposed to the medical community for therapy planning. This may allow interventional physicians to non-invasively predict the hemodynamic significance of renal artery stenosis. In the future, it may also provide physicians with diagnostic indicators and prognostic tools for the election of angioplasty and/or stenting, ideally even before the patient becomes symptomatic. Hence, CFD might provide the opportunity for improved therapeutic planning and potential screening for disturbances of cardiovascular hemodynamics. RAS screening and proper patient selection could become a reality based on a careful and judicious risk-to-benefit analysis. Further research is necessary to examine a statistically adequate number of severe RAS cases, along with healthy and post-treatment cases, as well as to increase the accuracy of CFD modeling by optimizing geometry representation, mesh resolution, and computer aided design of stents and other intravascular devices.

\section{Acknowledgement}

This work was funded through the University of Patras Research Committee (K. Karatheodoris program). Additionally, the authors would like to thank Dr. Luca Antiga (Mario Negri Institute, Ranica, Italy) for his fruitful comments on the manuscript.

\section{Conflict of Interest Statement}

None 


\section{Captions to figures}

Fig. 1. Flow diagram representing surface reconstruction steps. (a) Initial acquired DICOM images, (b) three-dimensional volume, (c) thresholding application, (d) seeded region growing segmentation algorithm application, (e) export to STL file, (f) geometry imported in the CFD software.

Fig. 2. STL segmentation data as obtained before treatment. (a) Arrows focus on renal artery segment, and (b) Zoomed, arrows point to atheromatic plaque segment.

Fig. 3. Surface mesh resolution near plaque segment.

Fig. 4. Arterial wall surface and WSS vector plots (reduced) on renal artery segment at peak pulse conditions, for (a, b) Case 1, (c, d) Case 2 and (e, f) Case 3. (a, c, e) Flow across the whole renal artery, and (b, d, f) Focused on arterial plaque zone near flow entrance.

Fig. 5. Streamlines within renal artery segment at peak pulse conditions, for (a, b) Case 1, (c, d) Case 2 and (e, f) Case 3. (a, c, e) Flow across the whole renal artery, and (b, d, f) Focused on arterial plaque zone near flow entrance.

Fig. 6. Flow rate as a function of time, in renal arterial segment under different conditions. Case 1: atheromatic plaque developed and present. Case 2: stent implanted and no plaque present. Case 3: originally healthy patient.

Fig. 7. Evolution of average and maximum differential pressure between inlet and outlet sections of renal arterial segment under various conditions. (a) Case 1: plaque developed and present. (b) Case 2: stent implanted and no plaque present. (c) Case 3: originally healthy patient. (d) Comparison between average differential pressures between inlet and outlet section, for renal arterial segment with plaque present (Case 1), with stent present (Case 2), and of originally healthy patient (Case 3 ).

Fig. 8. Average and maximum Wall Shear Stress at plaque area as a function of time, for renal arterial segment under various conditions. (a) Case 1: plaque developed and present. (b) Case 2: stent implanted and no plaque present. (c) Case 3: originally healthy patient. (d) Comparison of average Wall Shear Stresses at plaque section for arterial segment between 
different conditions. Condition with plaque present (Case 1), stent present (Case 2), and originally healthy patient (Case 3).

Fig. 9. Internal pressure and pressure gradient distributions along major local axis $\mathrm{X}$, for renal arterial segment under various conditions. (a) Case 1: plaque developed and present. (b) Case 2: stent implanted and no plaque present. (c) Case 3: originally healthy patient.

Fig. 10. Reconstructed and simulated ideal arterial constriction along with the adjacent aorta. Geometric and physical data identical to the work of Yim et al [4]. Streamlines at time $=0.3 \mathrm{~s}$.

Fig. 11. Differential pressure in a model constricted renal segment. Comparison between the present work and experimental and simulation results of Yim et al [4].

Fig. 12. (a) Domain walls and (b) internal streamlines in a renal aortic and adjoin right (R) and left (L) arterial segment using turbulent SST description (Case B BCs applied in aorta inlet). 


\section{References}

[1]. Harding MB, Smith LR, Himmelstein SI, Harrison K, Phillips HR, Schwab SJ, Hermiller JB, Davidson CJ, Bashore TM. Renal artery stenosis: prevalence and associated risk factors in patients undergoing routine cardiac catheterization. J Am Soc Nephrol 1992;2(11):1608-16.

[2]. Rundback JH, Sacks D, Kent KC, Cooper C, Jones D, Murphy T, Rosenfield K, White C, Bettmann M, Cortell S, Puschett J, Clair D, Cole P. Guidelines for the reporting of renal artery revascularization in clinical trials. American Heart Association. Circulation 2002;106(12):1572-85.

[3]. Rundback JH, Sacks D, Kent KC, Cooper C, Jones D, Murphy T, Rosenfield K, White C, Bettmann M, Cortell S, Puschett J, Clair DG, Cole P. Guidelines for the reporting of renal artery revascularization in clinical trials. J Vasc Interv Radiol 2003;14(9 Pt 2):S477-92.

[4]. Yim PJ, Cebral JR, Weaver A, Lutz RJ, Soto O, Vasbinder GB, Ho VB, Choyke PL. Estimation of the differential pressure at renal artery stenoses. Magn Reson Med 2004;51(5):969-77.

[5]. Martin LG, Rundback JH, Sacks D, Cardella JF, Rees CR, Matsumoto AH, Meranze SG, Schwartzberg MS, Silverstein MI, Lewis CA. Quality improvement guidelines for angiography, angioplasty, and stent placement in the diagnosis and treatment of renal artery stenosis in adults. J Vasc Interv Radiol 2003;14(9 Pt 2):S297-310.

[6]. Rees CR. Stents for atherosclerotic renovascular disease. J Vasc Interv Radiol 1999;10(6):689-705.

[7]. Varghese SS,Frankel SH. Numerical modeling of pulsatile turbulent flow in stenotic vessels. J Biomech Eng 2003;125(4):445-60.

[8]. Younis BA,Berger SA. A turbulence model for pulsatile arterial flows. Journal of Biomechanical Engineering-Transactions of the Asme 2004;126(5):578-84.

[9]. Ojha M, Cobbold RSC, Johnston KW, Hummel RL. Pulsatile flow through constricted tubes an experimental investigation using photochromic tracer methods. J Fluid Mech 1989;203:173-97.

[10]. Wentzel JJ, Krams R, Schuurbiers JC, Oomen JA, Kloet J, van Der Giessen WJ, Serruys PW, Slager CJ. Relationship between neointimal thickness and shear stress after Wallstent implantation in human coronary arteries. Circulation 2001;103(13):1740-5.

[11]. Steinman DA. Image-based computational fluid dynamics modeling in realistic arterial geometries. Ann Biomed Eng 2002;30(4):483-97.

[12]. Carlier SG, van Damme LC, Blommerde CP, Wentzel JJ, van Langehove G, Verheye S, Kockx MM, Knaapen MW, Cheng C, Gijsen F, Duncker DJ, Stergiopulos N, Slager CJ, Serruys PW, Krams R. Augmentation of wall shear stress inhibits neointimal hyperplasia after stent implantation: inhibition through reduction of inflammation? Circulation 2003;107(21):2741-6.

[13]. Taylor CA,Draney MT. Experimental and computational methods in cardiovascular fluid mechanics. Ann Rev Fluid Mech 2004;36:197-231.

[14]. Wentzel JJ, Kloet J, Andhyiswara I, Oomen JA, Schuurbiers JC, de Smet BJ, Post MJ, de Kleijn D, Pasterkamp G, Borst C, Slager CJ, Krams R. Shear-stress and wall-stress regulation of vascular remodeling after balloon angioplasty: effect of matrix metalloproteinase inhibition. Circulation 2001;104(1):91-6.

[15]. Wentzel JJ, Gijsen FJ, Schuurbiers JC, Krams R, Serruys PW, De Feyter PJ, Slager CJ. Geometry guided data averaging enables the interpretation of shear stress related plaque development in human coronary arteries. J Biomech 2005;38(7):1551-5.

[16]. Ku DN. Blood flow in arteries. Ann Rev Fluid Mech 1997;29:399-434.

[17]. Taylor CA, Hughes TJ, Zarins CK. Finite element modeling of blood flow in arteries. Comput Method Appl M 1998;158:155-96.

[18]. Taylor CA, Draney MT, Ku JP, Parker D, Steele BN, Wang K, Zarins CK. Predictive medicine: computational techniques in therapeutic decision-making. Comput Aided Surg 1999;4(5):231-47.

[19]. Berger SA,Jou LD. Flows in stenotic vessels. Ann Rev Fluid Mech 2000;32:347-82.

[20]. Menter FR. Two-equation eddy-viscosity turbulence models for engineering applications. AIAA J 1994;32(8):1598-605.

[21]. Menter FR. A comparison of some recent eddy-viscosity turbulence models. J Fluids EngTrans ASME 1996;118(3):514-9.

[22]. Bardina JE, Huang PG, Coakley TJ. Turbulence modeling validation. in 28th Fluid dynamics conference. 1997. Snowmass Village, CO: AIAA. 
[23]. Algorri M,Schmitt F. Reconstructing the surface of unstructured 3D data. in SPIE Medical Imaging. 1995.

[24]. AnalyzeDirect, AnalyzeDirect, in Visualization and Analysis software manual, BIR, Mayo Clinic.

[25]. Boutsianis E, Dave H, Frauenfelder T, Poulikakos D, Wildermuth S, Turina M, Ventikos Y, Zund G. Computational simulation of intracoronary flow based on real coronary geometry. Eur J Cardiothorac Surg 2004;26(2):248-56.

[26]. Lorensen WE,Cline HE. Marching cubes: a high resolution 3-D surface construction algorithm. Comp Graph 1987;12(3):163-9.

[27]. Bulpitt AJ,Berry E. Spiral CT of abdominal aneurysms: comparison of segmentation with an automatic 3D deformable model and interactive segmentation. in SPIE Medical Imaging. 1998.

[28]. Prakash S,Ethier CR. Requirements for mesh resolution in 3D computational hemodynamics. J Biomech Eng 2001;123(2):134-44.

[29]. Jin S, Oshinski J, Giddens DP. Effects of wall motion and compliance on flow patterns in the ascending aorta. J Biomech Eng 2003;125(3):347-54.

[30]. Hassan T, Timofeev EV, Saito T, Shimizu H, Ezura M, Tominaga T, Takahashi A, Takayama K. Computational replicas: anatomic reconstructions of cerebral vessels as volume numerical grids at three-dimensional angiography. AJNR Am J Neuroradiol 2004;25(8):1356-65.

[31]. Morris L, Delassus P, Walsh M, McGloughlin T. A mathematical model to predict the in vivo pulsatile drag forces acting on bifurcated stent grafts used in endovascular treatment of abdominal aortic aneurysms (AAA). J Biomech 2004;37(7):1087-95.

[32]. Yaras MI,Grosvenor AD. Evaluation of one- and two-equation low-Re turbulence models. Part I - Axisymmetric separating and swirling flows. Int J Numer Meth Fl 2003;42:1293-319.

[33]. Yaras MI,Grosvenor AD. Evaluation of one- and two-equation low-Re turbulence models. Part II - Vortex-generator jet and diffusing S-duct flows. Int J Numer Meth Fl 2003;42:132143.

[34]. CFX A, Ansys CFX, in Computational Fluid Dynamics software manuals, Ansys Inc.

[35]. Taylor CA, Hughes TJ, Zarins CK. Finite element modeling of three-dimensional pulsatile flow in the abdominal aorta: relevance to atherosclerosis. Ann Biomed Eng 1998;26(6):97587.

[36]. Ryval J, Straatman AG, Steinman DA. Two-equation turbulence modeling of pulsatile flow in a stenosed tube. J Biomech Eng 2004;126(5):625-35.

[37]. Zeller T. Renal artery stenosis: epidemiology, clinical manifestation, and percutaneous endovascular therapy. J Interv Cardiol 2005;18(6):497-506.

[38]. White CJ. Catheter-based therapy for atherosclerotic renal artery stenosis. Circulation 2006;113(11):1464-73.

[39]. Safian RD,Textor SC. Renal-artery stenosis. N Engl J Med 2001;344(6):431-42.

[40]. Wentzel JJ, Gijsen FJ, Stergiopulos N, Serruys PW, Slager CJ, Krams R. Shear stress, vascular remodeling and neointimal formation. J Biomech 2003;36(5):681-8.

[41]. Wentzel JJ, Janssen E, Vos J, Schuurbiers JC, Krams R, Serruys PW, de Feyter PJ, Slager CJ. Extension of increased atherosclerotic wall thickness into high shear stress regions is associated with loss of compensatory remodeling. Circulation 2003;108(1):17-23.

[42]. Bathe M,Kamm RD. A fluid--structure interaction finite element analysis of pulsatile blood flow through a compliant stenotic artery. J Biomech Eng 1999;121(4):361-9.

[43]. Tang D, Yang C, Huang Y, Ku DN. Wall stress and strain analysis using a three-dimensional thick-wall model with fluid-structure interactions for blood flow in carotid arteries with stenoses. Comput Struct 1999;72:341-56.

[44]. Tang D, Yang C, Ku DN. A 3-D thin-wall model with fluid-structure interactions for blood flow in carotid arteries with symmetric and asymmetric stenoses. Comput Struct 1999;72:35777.

[45]. Tang D, Yang J, Yang C, Ku DN. A nonlinear axisymmetric model with fluid-wall interactions for steady viscous flow in stenotic elastic tubes. J Biomech Eng 1999;121:494501.

[46]. Qiu Y,Tarbell JM. Interaction between wall shear stress and circumferential strain affects endothelial cell biochemical production. J Vasc Res 2000;37(3):147-57.

[47]. Buchanan JR, Kleinstreuer C, Comer JK. Rheological effects on pulsatile hemodynamics in a stenosed tube. Comput Fluids 2000;29:695-724. 
[48]. Leertouwer TC, Gussenhoven EJ, Bosch JL, van Jaarsveld BC, van Dijk LC, Deinum J, Man In 't Veld AJ. Stent placement for renal arterial stenosis: where do we stand? A meta-analysis. Radiology 2000;216(1):78-85. 